\title{
The effects of GelMA hydrogel on nerve repair and regeneration in mice with spinal cord injury
}

\author{
Hongcheng Zhang ${ }^{1}$, Jinhui $\mathrm{Xu}^{2}$, Saijilafu, ${ }^{1,3}$ \\ ${ }^{1}$ Department of Orthopaedics, the First Affiliated Hospital of Soochow University, Suzhou, China; ${ }^{2}$ Department of Orthopedic Oncology, \\ Changzheng Hospital, Shanghai, China; ${ }^{3}$ Orthopaedic Institute, Medical College, Soochow University, Suzhou, China \\ Contributions: (I) Conception and design: H Zhang, J Xu; (II) Administrative support: H Zhang; (III) Provision of study materials or patients: H \\ Zhang; (IV) Collection and assembly of data: All authors; (V) Data analysis and interpretation: H Zhang, Saijilafu; (VI) Manuscript writing: All \\ authors; (VII) Final approval of manuscript: All authors. \\ Correspondence to: Saijilafu. Department of Orthopaedics, the First Affiliated Hospital of Soochow University, No. 188 Shizi Street, Gusu District, \\ Suzhou 215006, China. Email: saijilafu2020@126.com.
}

Background: To determine the effects of gelatin methacryloyl (GelMA) hydrogel on nerve repair and regeneration in mice with spinal cord injury (SCI).

Methods: A total of 30 ICR mice (6-8 weeks old) were randomly assigned into the control group, the model group, and the experimental group via the random digits table method. There were 10 mice in each group. All mice underwent a T8 laminectomy. For mice in the experimental group and the model group, after the T8 laminectomy, SCI models were constructed by clamping the mice spinal cord tissue for 1 minute using an aneurysm clip (25 g). Additionally, the SCI area of each mouse in the experimental group was locally injected with $0.05-0.7 \mathrm{~mL}$ GelMA hydrogel $[10 \%(\mathrm{w} / \mathrm{v})]$ and photocrosslinking was initiated under a blue light source with a wavelength of $405 \mathrm{~nm}$. The exercise performance of each mouse was tested via the bedside mobility scale (BMS) on post-operative days 1, 3, 7, and 14. After 14 days, mice were sacrificed and the dorsal root ganglion (DRG) sensory neurons were isolated and cultured for 3 days in vitro. The axon lengths of the neurons were then evaluated. Immunohistochemical staining was performed to assess the development of syringomyelia in the area. Western blots (WB) and immunofluorescence staining were performed to quantify the expression of glial fibrillary acidic protein (GFAP), growth associated protein (GAP)43, and nestin in the DRG neurons from each group of mice.

Results: Compared with mice in the control group, mice in the SCI model group showed a notable decrease in exercise ability, while the exercise ability of mice in the experimental group recovered markedly after treatment with GelMA hydrogel. Administration of GelMA hydrogel lengthened the axon of DRG neurons in mice and reduced the area of syringomyelia. Furthermore, GelMA hydrogel inhibited scar formation and promoted the recovery of neurological function by upregulating GAP43 and nestin expression and downregulating GFAP expression.

Conclusions: In mice with SCI, local injection of GelMA hydrogel strongly inhibited scar formation, reduced the area of syringomyelia, and promoted nerve regeneration and recovery of limb movement function.

Keywords: GelMA hydrogel spinal cord injury (GelMA hydrogel SCI); nerve repair; regeneration; effect study

Submitted Apr 22, 2021. Accepted for publication Jul 15, 2021.

doi: 10.21037/atm-21-2874

View this article at: https://dx.doi.org/10.21037/atm-21-2874 


\section{Introduction}

Spinal cord injury (SCI) is a severe disease of the central nervous system (CNS) manifesting as movement and sensory dysfunction of various degrees and segments due to autonomic nervous system dysfunction (1). Not only does SCI cause physical and sensory damage to patients, but it can also lead to severe anxiety and depression, giving rise to an increased risk of suicide and decreased quality of life (2). Unfortunately, the CNS has a limited ability for spontaneous regeneration. After nerve injury, activated glial cells secrete inhibitory factors that prevent the growth of the myelin sheath and neurons, and dense scar tissues usually form around the injury site (3). Currently, surgical stabilization is the main treatment for SCI, followed by rehabilitative nursing support (4). Despite rehabilitation, function recovery is generally very limited, and thus, the recovery of nerve regeneration after SCI has become a focal point in scientific research and clinical treatment.

Gelatin methacryloyl (GelMA) hydrogels possess highly desirable physical and biological properties (5). Certain cell attachment motifs and matrix metalloproteinase reactive peptide motifs have been diffused well into the GelMA hydrogel scaffold, and these encourage cell proliferate. With such characteristics, GelMA hydrogel is highly similar to some natural extracellular matrixes with important characteristics (6). GelMA hydrogel can be crosslinked in one minute or even a few seconds with a photoinitiator with a relatively low concentration (7). It can promote cell-to-cell interaction, migration, and more effective metabolism (8). Dursun Usal and colleagues (9) demonstrated that GelMA-pHEMA hydrogel improved the mechanical strength needed for suture of guide wires as a nerve conduit, and it contributed to ideal nerve guidance in the treatment of peripheral nerve injury. Additionally, Ning et al. (10) showed that GelMA hydrogelprolonged the release of peptides which promoted bone healing in rats. Zhou et al. (11) implant the functional GelMA hydrogel scaffold loaded with BMSCs and NSCs into the hemicomsected spinal cord of rats can significantly promote motor function recovery and neuronal differentiation, and reduce glial scar, fibrotic scar and inflammatory response. However, there are few reports on the effects of direct injection of Gelma on nerve repair and regeneration in mice with spinal cord injury. So this study constructed mice models of SCI and examined the effects of GelMA hydrogel on nerve repair and regeneration in mice with SCI.
We present the following article in accordance with the ARRIVE reporting checklist (available at https://dx.doi. org/10.21037/atm-21-2874).

\section{Methods}

\section{Experimental animals}

A total of 30 ICR mice (6-8 weeks old, 200-300 g, male or female) were provided by the Center of Animal Laboratory, Soochow University (Suzhou, China). Mice were housed for 1 week in a clean environment with humidity of $40-70 \%$ and temperature of $20-26^{\circ} \mathrm{C}$, and provided free access to food and water. The protocol was prepared before the study without registration. This study and all included experimental protocols and surgical procedures were approved by the Ethics Committees of the First Affiliated Hospital of Soochow University and the Animal Ethics Committee at Soochow University, China (approval No. SUDA20200316A01). All experiments were conducted in accordance with the Guidelines for the Care and Use of Laboratory Animals (12).

\section{Preparation of GelMA hydrogel}

Gelatin was dissolved in phosphate buffered saline (PBS) at $50{ }^{\circ} \mathrm{C}(\mathrm{pH} 7.4)$, followed by addition of methacrylic anhydride. The reaction was terminated by addition of PBS (5 times the volume of gelatin). The solution was then dialyzed in deionized water for 5-7 days through a 12-14 kDa dialysis bag to eliminate small molecular impurities. The deionized water was replaced twice a day. The dialyzed GelMA solution was freeze-dried or stored at low temperature.

\section{Establishment of the SCI model in mice}

Briefly, 30 mice were randomly divided into control group, model group and experimental group by random digits table method. There were 10 mice in each group. Specific steps: 30 mice were numbered $01-30$, and the starting point 01 and the end point 30 of sampling were specified on the random number table. The first 10 numbers on the random number list were divided into the control group, the last 10 numbers into the model group, and the remaining 10 numbers into the experimental group. The SCI model was established in model group $(\mathrm{n}=10)$ and experimental 


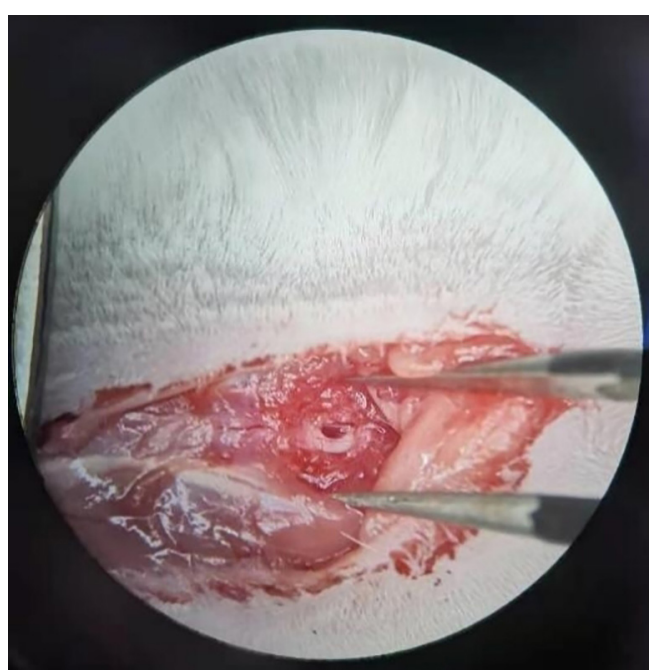

Figure 1 Under the microscope, the spinal cord was completely removed by hemicomsection.

group ( $\mathrm{n}=10)$ mice as previously described (13). Mice were anesthetized by intraperitoneal injection of xylazine $(10 \mathrm{mg} / \mathrm{kg})$ and ketamine $(100 \mathrm{mg} / \mathrm{kg})$, and the back hairs were removed. The mice were fixed on the operating table in a prone position, disinfected with $75 \%$ ethanol, and a middle dorsal incision was made. The skin and subcutaneous tissues were cut open successively by approximately $2 \mathrm{~cm}$ with T8 at the center. The paraspinal muscles were sharply cut and separated to either side, exposing the spinous process and lamina of the T8-L1 vertebral body. The spinous process and whole lamina of the T9-T11 vertebral body were removed with a mosquito clamp. By counting the ribs, it was found that the spinous process of $\mathrm{T} 9$ was oblique to the caudal side, T11 was oblique to the cephalic side, and T10 was neutral. With T10 as the center, the spinal cord tissue was clamped for 1 minute with an aneurysm clip $(25 \mathrm{~g})$, thereby causing SCI (Figure 1). All the 20 mice were successfully modeled. For mice in the experimental group $(n=10)$, prepared GelMA hydrogel was implanted into the injury site of each mouse, and the muscles and skin were sutured. For mice in the model group $(n=10)$, the muscles and skin were sutured without any other intervention. For the control group $(n=10)$, the spinal cord was exposed during the modeling, but these mice were not subjected to clamping with the aneurysm clip. If the mice had severe adverse reactions during the experiment or were on the verge of death, they were killed by cervical dislocation.

\section{Outcome measures}

Behavioral scoring with the bedside mobility scale (BMS) (14)

The total score and sub-score of the BMS were adopted to evaluate the exercise performance of mice on post-operative days $1,3,7$, and 14 . The BMS score ranges from 0 to 9 points, and the maximum sub-score is 11 points. Higher scores indicate better exercise movement function of the mouse.

\section{Comparison of the axon length of neurons}

At 14 days, mice in the model group $(n=10)$ and the experimental group $(\mathrm{n}=10)$ were sacrificed via intraperitoneal anesthesia. The dorsal root ganglion (DRG) tissues were dissected and incubated in collagenase A $(1.0 \mathrm{mg} / \mathrm{mL})$ at $37^{\circ} \mathrm{C}$ for 90 minutes. The samples were then digested with $0.25 \%$ trypsin at $37{ }^{\circ} \mathrm{C}$ for 15 minutes. Minimal essential medium (MEM) containing $10.0 \%$ fetal bovine serum (FBS) was added to terminate the digestive process. The isolated DRG sensory neurons were placed on coverslips and cultured in MEM containing $5 \% \mathrm{FBS}$, $10 \mathrm{mg} / \mathrm{mL}$ streptomycin, and $100 \mathrm{IU} / \mathrm{mL}$ penicillin for 3 days at $37{ }^{\circ} \mathrm{C}$ and $5 \% \mathrm{CO}_{2}$ in an incubator (Thermo Fisher Scientific, Waltham, MA, USA).

\section{Immunofluorescence staining of the DRG}

To determine the nuclear localization of BCL2L11, the neurons from the SCI model were treated with tubulin tyrosine ligase (TTL) for 1 hour followed by washing with PBS. Cells were then fixed in $4 \%$ paraformaldehyde for 30 minutes, washed, and permeabilized in cold acetone for 30 minutes. Thereafter, the cells were sealed in $5 \%$ bovine serum albumin (BSA) (Hengfei Biotechnology Co., Ltd., Shanghai, China, R00911) containing $0.5 \%$ goat serum for 1 hour, and incubated with a BCL2L11 primary antibody for 1 hour at room temperature. Finally, the cells were washed with PBS and incubated in a FITC-conjugated secondary antibody for 45 minutes at room temperature in the dark.

\section{Area of syringomyelia}

After 14 days, mice were sacrificed and the original surgical incision was opened. The spinal cord tissues ( $2 \mathrm{~cm}$ around the injured site) were harvested, immobilized in $40 \mathrm{~g} / \mathrm{L}$ paraformaldehyde for 48 hours, followed by 
freezing and sectioning (longitudinal sectioning, $10 \mu \mathrm{m}$ ). Immunofluorescence staining was performed on the sections. Briefly, the sections were incubated with $\mathrm{Cdk} 5$, SYN, or SNARE antibodies (dilution ratio of 1:200) at $4{ }^{\circ} \mathrm{C}$ overnight, followed by a goat anti-rabbit secondary antibody (dilution ratio of $1: 200)$ at $4{ }^{\circ} \mathrm{C}$ overnight. All antibodies were purchased from Shenzhen OTWO Biotechnology Co., Ltd. The syringomyelia after SCI was evaluated under a Leica confocal scanning microscope.

\section{Western blot (WB)}

Spinal cord tissues $(50 \mathrm{mg})$ were lysed in $500 \mu \mathrm{L}$ lysis solution, followed by homogenization on an ice bath and centrifugation for 20 minutes $\left(12,000 \mathrm{~g}, 4^{\circ} \mathrm{C}\right)$ to collect the supernatant. The protein concentration of the supernatant was quantified using the BCA kit (Fantai Biotechnology Co., Ltd., Shanghai, CN, FT-D24505), and the protein were isolated on a $12 \%$ SDS-PAGE (sodium dodecyl-sulfate polyacrylamide gel electrophoresis; Yiyan Biotechnology Co. Ltd., Shanghai, China, EY-0975) and transferred ontopolyvinylidene fluoride (PVDF) membranes. Membranes were blocked in $5 \%$ skim milk followed by incubation with the primary antibody $(1: 1,000)$ overnight at $4{ }^{\circ} \mathrm{C}$. After washing with PBS, the membrane was incubated with horseradish peroxidase labeled goat anti-rabbit secondary antibody $(1: 1,000)$ at $37{ }^{\circ} \mathrm{C}$ for 1 hour, and rinsed thoroughly 3 times with PBS for 5 minutes each time. Protein bands were visualized with an electrochemiluminescence (ECL) agent and images were captured using the Quantity One infrared imaging system. The relative expression of proteins was recorded as the gray value of the band and compared to that of an internal reference protein band, namely, b-actin.

\section{Determination of the optical density (OP) of glial fibrillary acidic protein (GFAP)}

The spinal cord sections from mice in the experimental group and the model group were examined at $400 \times$ magnification. Pictures were taken along the periphery of the scar to ensure the coverage of the injured area. Semiquantitative analysis was carried out on all collected images with NIS-Elements $\mathrm{Br} 3.0$, and the average OP of GFAP was determined directly. The arithmetic mean $\mathrm{OP}$ of each specimen was analyzed and compared.

\section{Statistical analyses}

The GraphPad 6 software was used for all data analyses and preparation of figures. If the data fit the normal distribution, Inter-group comparisons and multi-group comparisons of all data, presented as the mean \pm standard deviation (SD), were carried out via independent-sample $t$-tests and one-way analysis of variance (ANOVA; expressed by F), respectively. Post hoc pairwise comparisons were carried out via the Fishers LSD-t-test. Expression at multiple time points was analyzed via the repeated measures analysis of variance, and expressed as $\mathrm{F}$, and Bonferroni post hoc tests were carried out. If the data were not normally distributed, the median (P25, P75) was used to represent the data. Intergroup comparison and multi-group comparison were performed by Mann-Whitney U test and Kruskal-Wallis test. A P value $<0.05$ was considered statistically significant. The SPSS25.0 software from IBM company was used for data analyses.

\section{Results}

A comparison of bedside motility scale (BMS) scores among the three groups

Compared to mice in the control group, mice that underwent SCI in the model group and the experimental group had notably lower total scores and sub-scores on the BMS on the first day after surgery. These scores significantly increased 3 days after SCI. On post-operative days 3,7 , and 14 , the experimental group showed markedly higher total scores and sub-scores compared to the model group $(\mathrm{P}<0.05$; Figure 2), suggesting that intervened with GelMA hydrogel improved exercise ability in SCI mice.

\section{A comparison of axon length of the neurons after intervention with GelMA hydrogel}

The axon length of neurons isolated from mice in the experimental group $(161 \pm 9.3)$ who were treated with GelMA hydrogel were significantly longer than the neurons isolated from mice in the model group $(100 \pm 5.1)(\mathrm{P}<0.05$; Figure 3).

\section{Amelioration of the area of syringomyelia after intervention with GelMA hydrogel}

Mice in the experimental group $(2.3 \pm 0.1)$ showed a significantly decreased area of syringomyelia after treatment with GelMA hydrogel, compared with the model group $(4.5 \pm 0.2)(\mathrm{P}<0.05$; Figure 4). 
A

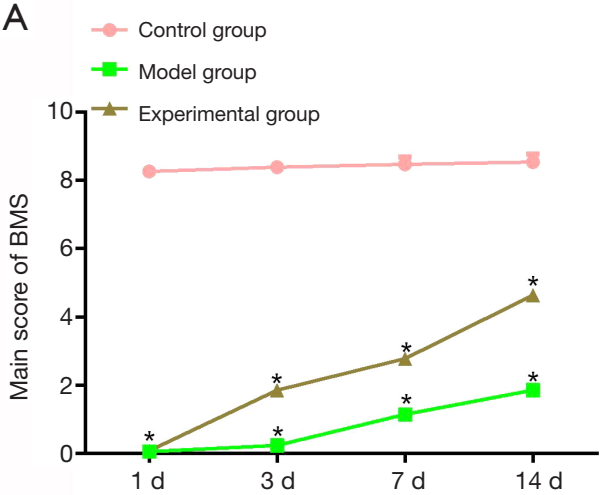

B

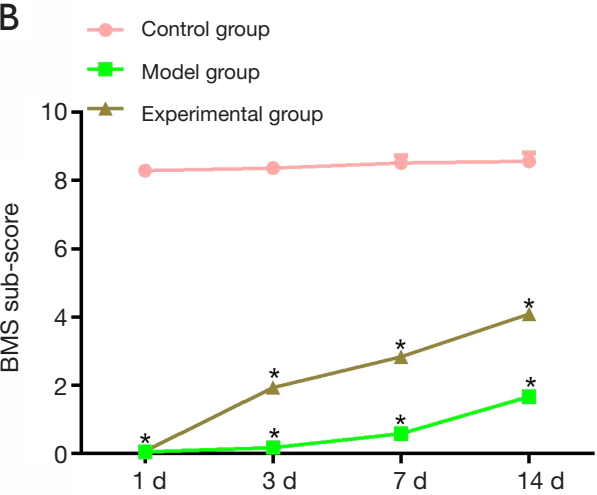

Figure 2 A comparison of bedside motility scale (BMS) scores among the three groups at different time points after intervention. (A) The effects of GelMA hydrogel on the total BMS score in mice with spinal cord injury (SCI). (B) The effects of GelMA hydrogel on the BMS sub-score in mice with SCI. Comparison with the control group or inter-group comparison, ${ }^{*}, \mathrm{P}<0.05$.

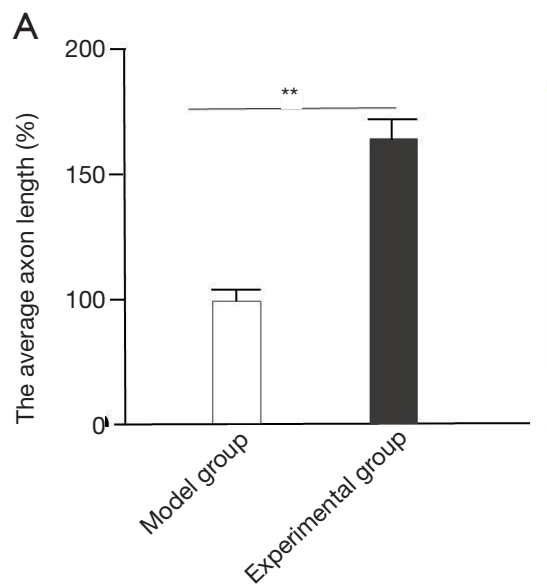

B
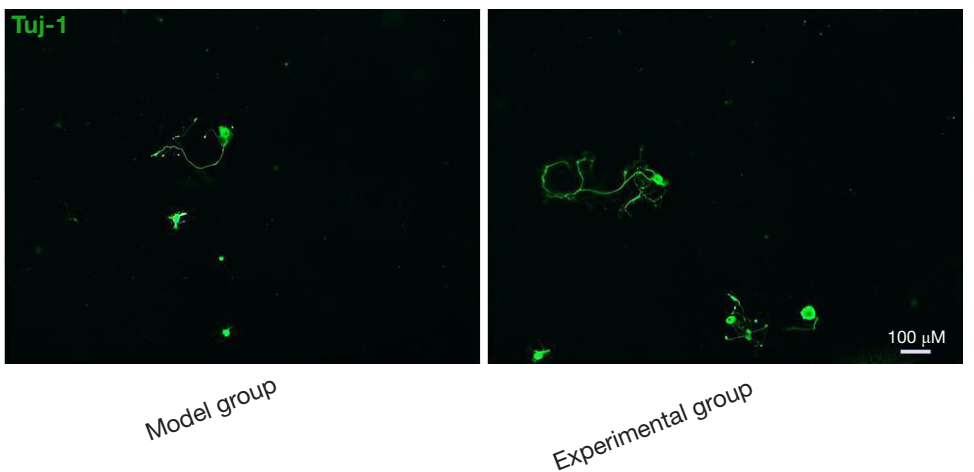

Figure 3 A comparison of the axon length of neurons after intervention with GelMA hydrogel. (A) The effect of GelMA hydrogel on the axon length of neurons in mice with spinal cord injury (SCI). (B) The growth of the axon of neurons after treatment with GelMA hydrogel. Comparison with the control group or in inter-group comparison, ${ }^{* *}, \mathrm{P}<0.01$.

\section{The effects of GelMA hydrogel on nerve repair and regeneration}

Mice in the model group showed significantly increased expression of growth associated protein (GAP)43, nestin, and GFAP compared with mice in the control group (all $\mathrm{P}<0.05$ ). In the experimental group, intervention with GelMA hydrogel significantly increased GAP43 and nestin expression, but decreased GFAP expression (all $\mathrm{P}<0.05$; Figure 5).

\section{The effects of GelMA bydrogel on the OP of GFAP}

Compared with mice in the control group, mice in the model group showed notably increased OP of GFAP $(\mathrm{P}<0.05)$, and after intervention with GelMA hydrogel, the experimental group showed significantly decreased OP of GFAP $(\mathrm{P}<0.05$; Figure 6).

\section{Discussion}

SCI often severely damages a patient's movement, sensory, and autonomic neurological functions, resulting in significant emotional and financial burden (15). SCI often results in activation of astrocytes in large quantities (16), which inhibits the expression of nutritional factors, and also inhibits axon growth-associated proteins, 


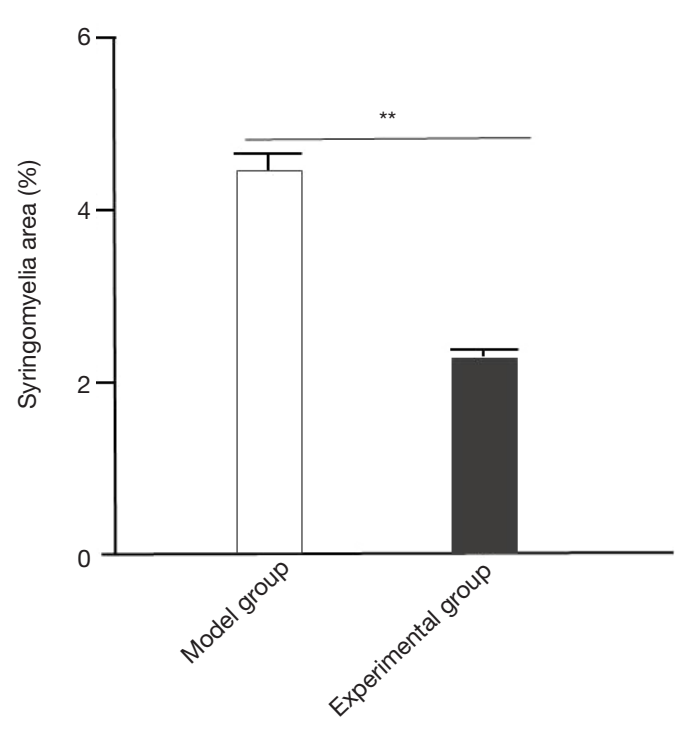

Figure 4 Amelioration of the area of syringomyelia after intervention with GelMA hydrogel. The effects of GelMA hydrogel on the area of syringomyelia in mice with spinal cord injury (SCI). Comparison with the control group or in inter-group comparison, ${ }^{* *}, \mathrm{P}<0.01$.

thereby hindering the repair and regeneration of damaged neurons (17). Therefore, effectively repairing SCI is of crucial importance.

This study demonstrated that after intervention with GelMA hydrogel, mice with SCI presented significantly better movement function as well as improved nerve repair and regeneration abilities. The recovery of limb movement function after SCI depends on axonal regeneration of injured nerve cells (18). Implantation of a hydrogel scaffold may be beneficial in patients with SCI. Hydrogel has been shown to be nontoxic to cells, have a strong antiregeneration effect on bone marrow mesenchymal stem cells and macrophages, and contribute to fast recovery of limb movement function in model animals (19). The conclusions of this latter study were similar to this current investigation. Compared with the control group, the spinal cord injured mice showed significantly lower total scores and sub-scores on the BMS on post-operative day 1. These scores significantly increased 3 days after SCI. Additionally, on post-operative days 3,7 , and 14 , the experimental group that had been treated with GelMA hydrogel achieved significantly higher total scores and sub-scores on the BMS compared to the model group. These results suggested that intervention with GelMA hydrogel improved the scored on the BMS and restored limb movement function in SCI mice. Studies have suggested that it is very difficult to grow neuronal processes after SCI (20). As hydrogel is capable of transferring genes, it provides a combined method for nerve regeneration. Not only can hydrogel support the growth of neurites and gene transfer, but it can also induce the expression of inducible factors. Furthermore, studies have shown that neurites produced by the delivery of lipid complexes in the gel are longer (21). These latter results agree with this current report which demonstrated that compared with the model group, the experimental group showed significantly increased axon length of neurons after treatment with GelMA hydrogel. This suggested that GelMA hydrogel promoted the regeneration of neuronal axons after SCI. This is further supported by the report by Fan et al., showing that intervention with GelMA hydrogel promoted nerve regeneration in cases with SCI, reduced the cavity area of mice, strongly promoted function recovery, inhibited the formation of glial scars, and accelerated axonal regeneration (22). In our study, in contrast to the model group, the experimental group showed significantly decreased area of syringomyelia after treatment with GelMA hydrogel, suggesting that intervention with GelMA hydrogel contributed to superior repair of the spinal cord and better alleviation of internal bleeding, tissue edema, and cavitation of spinal cord structure after injury.

GFAP is a specific protein found in astrocytes and can be used to measure the proliferation of astrocytes (23). Nestin is an intermediate filament protein peculiar to neural stem cells (24). GAP43 regulates neuronal growth and axonal regeneration in normal nerve tissues (25). Therefore, these three proteins were used as markers of nerve injury to evaluate the beneficial effects of GelMA hydrogel on spinal nerve trauma. According to a study by $\mathrm{Li}$ and colleagues (26), hydrogel can improve the abundance of GAP43 and GFAP in cell fibers. Our current study demonstrated that expression of GAP43, nestin, and GFAP were significantly increased in the SCI mice compared to the control mice. However, in contrast to Li et al., while intervention with GelMA hydrogel significantly increased GAP43 and nestin, GFAP expression was significantly decreased. These results suggested that GelMA hydrogel can increase neural stem cells, promote their differentiation and growth, reduce the glial content in nerve cells, and can also reverse the nerve damage caused by reactive astrocytes. 
A

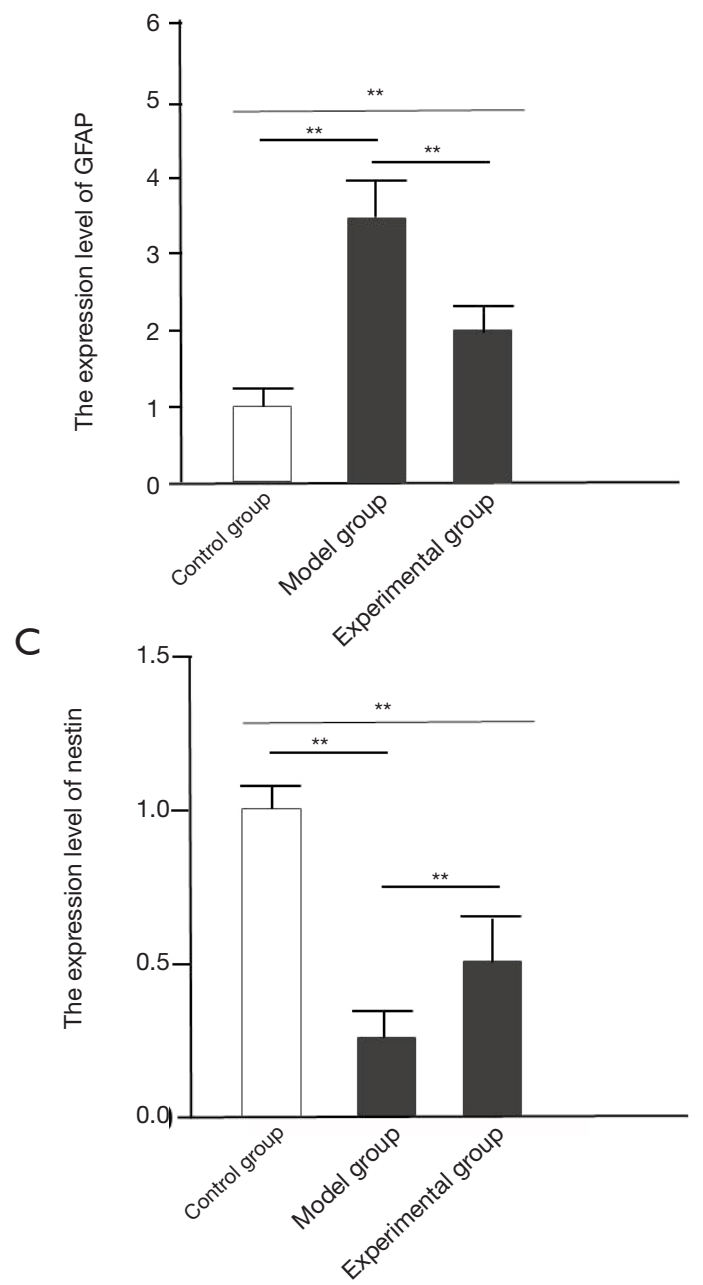

B
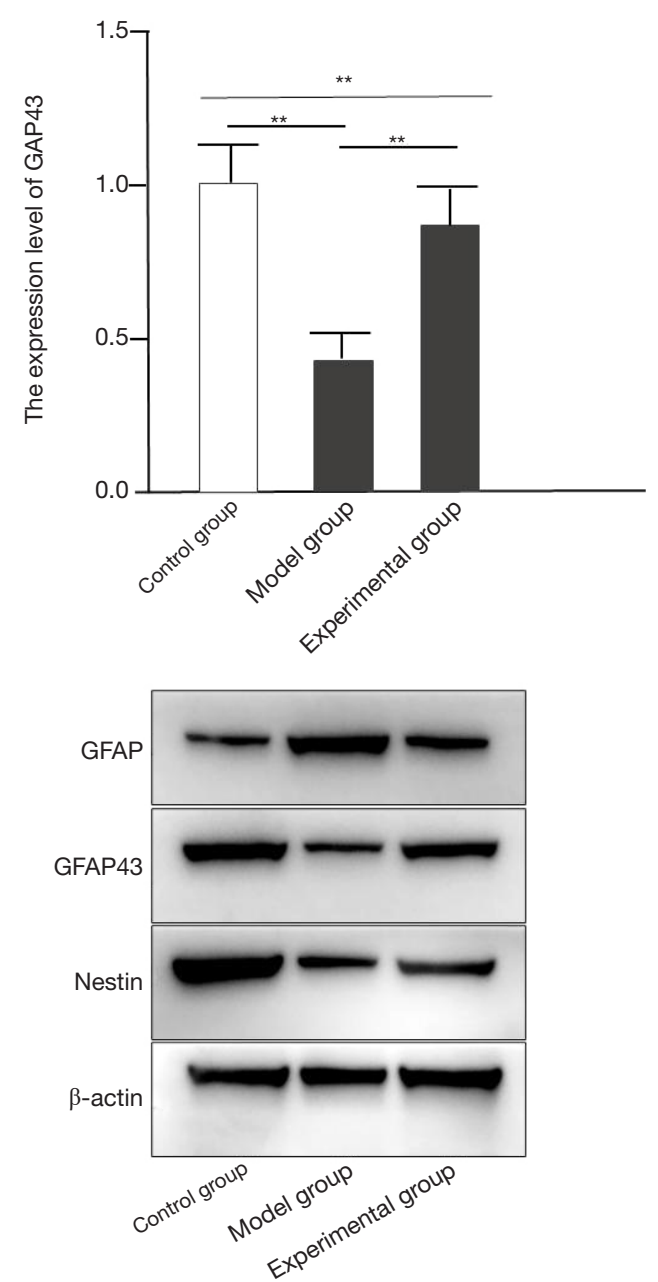

Figure 5 The effects of GelMA hydrogel on nerve repair and regeneration. (A) The effects of GelMA hydrogel on glial fibrillary acidic protein (GFAP) expression in mice with spinal cord injury (SCI). (B) The effects of GelMA hydrogel on growth associated protein (GAP) 43 expression in mice with SCI. (C) The effects of GelMA hydrogel on nestin protein expression in mice with SCI. (D) A representative Western blot showing the effects of GelMA hydrogel on protein expression of GFAP, GAP43, and nestin in mice with SCI. b-actin was used as the internal protein control. Comparison with the control group or in inter-group comparison, ${ }^{* *}, \mathrm{P}<0.01$.

The OP of GFAP in the model group was significantly increased compared to that in the control group. After intervention with GelMA hydrogel, the experimental group showed significantly decreased OP of GFAP, suggesting that GelMA hydrogel can cause weaker activation of astrocytes, prevent the formation of reactive astrocytes, and thus may be beneficial to the repair of the injured spinal cord. However, there are still some limitations in this study, such as whether GelMA has the same effect on other species.
Given that Gelma promotes nerve regeneration and limb motor function recovery in mice with spinal cord injury, we will further study the effects of Gelma on nerve repair and regeneration after spinal cord injury in other species to explain other species where Gelma may be applicable.

In summary, this investigation demonstrated that in SCI, local injection of GelMA hydrogel can inhibit scar formation, reduce the area of syringomyelia, and promote nerve regeneration and recovery of limb movement function. 
A

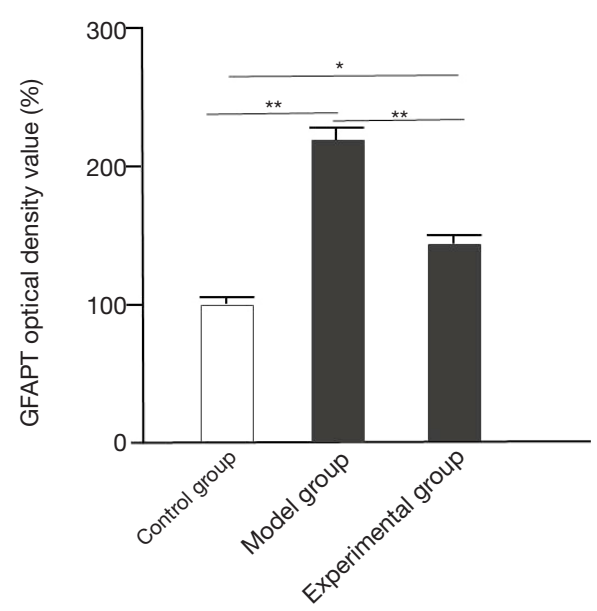

B
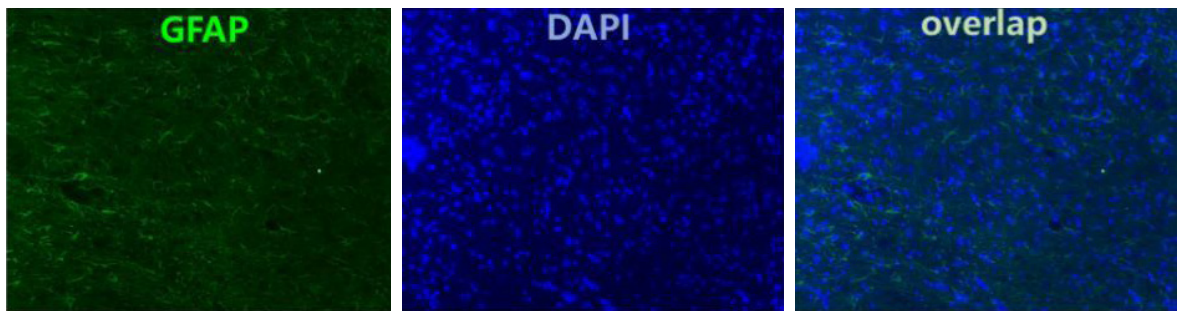

Control group

Model group
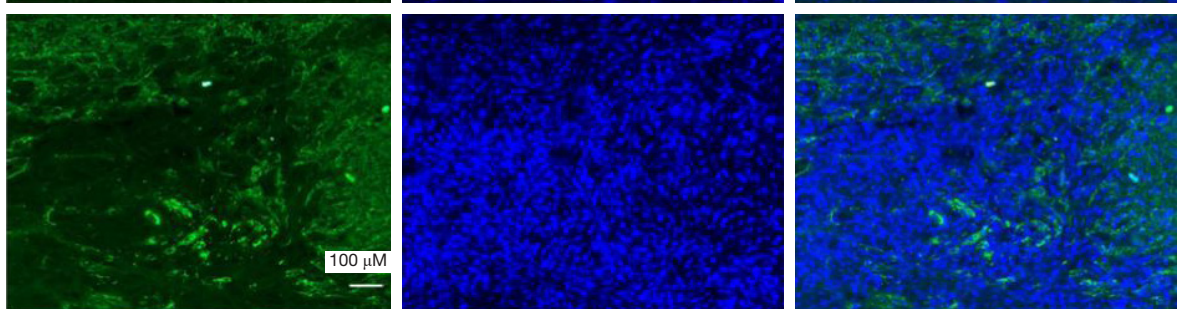

Experimental group
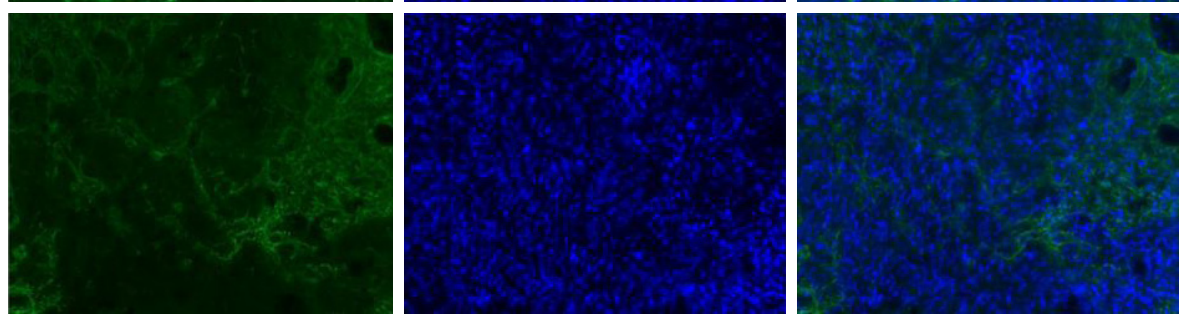

Figure 6 The effects of GelMA hydrogel on the optical density (OP) of glial fibrillary acidic protein (GFAP). (A) The effects of GelMA hydrogel on the OP of GFAP in mice with spinal cord injury (SCI). (B) A representative immunofluorescence stain showing the OP of GFAP in the spinal cord sections of control mice, SCI injury mice (model group), and SCI injury mice with GelMA hydrogel intervention (experimental group). Comparison with the control group or in inter-group comparison, * $\mathrm{P}<0.05$; **, $\mathrm{P}<0.01$.

\section{Acknowledgments}

Funding: This work was funded by the National Natural Science Foundation of China (Grant numbers 81571189 and 81772353).

\section{Footnote}

Reporting Checklist: The authors have completed the ARRIVE reporting checklist. Available at https://dx.doi. org/10.21037/atm-21-2874 
Data Sharing Statement: Available at https://dx.doi. org/10.21037/atm-21-2874

Conflicts of Interest: All authors have completed the ICMJE uniform disclosure form (available at https://dx.doi. org/10.21037/atm-21-2874). The authors have no conflicts of interest to declare.

Ethical Statement: The authors are accountable for all aspects of the work in ensuring that questions related to the accuracy or integrity of any part of the work are appropriately investigated and resolved. The protocol was prepared before the study without registration. This study and all included experimental protocols and surgical procedures were approved by the Ethics Committees of the First Affiliated Hospital of Soochow University and the Animal Ethics Committee at Soochow University, China (approval No.: SUDA20200316A01). All experiments were conducted in accordance with the Guidelines for the Care and Use of Laboratory Animals.

Open Access Statement: This is an Open Access article distributed in accordance with the Creative Commons Attribution-NonCommercial-NoDerivs 4.0 International License (CC BY-NC-ND 4.0), which permits the noncommercial replication and distribution of the article with the strict proviso that no changes or edits are made and the original work is properly cited (including links to both the formal publication through the relevant DOI and the license). See: https://creativecommons.org/licenses/by-nc-nd/4.0/.

\section{References}

1. Brown R, Burton AR, Macefield VG. Autonomic dysreflexia: Somatosympathetic and viscerosympathetic vasoconstrictor responses to innocuous and noxious sensory stimulation below lesion in human spinal cord injury. Auton Neurosci 2018;209:71-8.

2. Schmidt EKA, Torres-Espin A, Raposo PJF, et al. Fecal transplant prevents gut dysbiosis and anxiety-like behaviour after spinal cord injury in rats. PLoS One 2020;15:e0226128.

3. Zhang N, Chin JS, Chew SY. Localised non-viral delivery of nucleic acids for nerve regeneration in injured nervous systems. Exp Neurol 2019;319:112820.

4. Cheng XH, Zhang L, Fu J. Somatosensory evoked potential changes and decompression timing for spinal cord function recovery and evoked potentials in rats with spinal cord injury. Brain Res Bull 2019;146:7-11.

5. Rehman SRU, Augustine R, Zahid AA, et al. Reduced Graphene Oxide Incorporated GelMA Hydrogel Promotes Angiogenesis For Wound Healing Applications. Int J Nanomedicine 2019;14:9603-17.

6. Gan D, Xu T, Xing W, et al. Mussel-inspired dopamine oligomer intercalated tough and resilient gelatin methacryloyl (GelMA) hydrogels for cartilage regeneration. J Mater Chem B 2019;7:1716-25.

7. Li X, Wang Y, Xu F, et al. Artemisinin Loaded mPEG-PCL Nanoparticle Based Photosensitive Gelatin Methacrylate Hydrogels for the Treatment of Gentamicin Induced Hearing Loss. Int J Nanomedicine 2020;15:4591-606.

8. Xu W, Molino BZ, Cheng F, et al. On Low-Concentration Inks Formulated by Nanocellulose Assisted with Gelatin Methacrylate (GelMA) for 3D Printing toward Wound Healing Application. ACS Appl Mater Interfaces 2019;11:8838-48.

9. Dursun Usal T, Yucel D, Hasirci V. A novel GelMApHEMA hydrogel nerve guide for the treatment of peripheral nerve damages. Int J Biol Macromol 2019;121:699-706.

10. Ning Z, Tan B, Chen B, et al. Precisely Controlled Delivery of Abaloparatide through Injectable Hydrogel to Promote Bone Regeneration. Macromol Biosci 2019;19:e1900020.

11. Zhou P, Xu P, Guan J, et al. Promoting 3D neuronal differentiation in hydrogel for spinal cord regeneration. Colloids Surf B Biointerfaces 2020;194:111214.

12. Sikes RS, Animal Care and Use Committee of the American Society of Mammalogists. 2016 Guidelines of the American Society of Mammalogists for the use of wild mammals in research and education. J Mammal 2016;97:663-88.

13. Reinhardt DR, Stehlik KE, Satkunendrarajah K, et al. Bilateral cervical contusion spinal cord injury: A mouse model to evaluate sensorimotor function. Exp Neurol 2020;331:113381.

14. Bernhardt D, Konig L, Aufderstrasse S, et al. Generation of a New Disease-specific Prognostic Score for Patients With Brain Metastases From Small-cell Lung Cancer Treated With Whole Brain Radiotherapy (BMS-Score) and Validation of Two Other Indices. Clin Lung Cancer 2018;19:340-5.

15. Aimetti AA, Kirshblum S, Curt A, et al. Natural history of neurological improvement following complete (AIS A) thoracic spinal cord injury across three registries to guide 
acute clinical trial design and interpretation. Spinal Cord 2019;57:753-62.

16. Gong L, Lv Y, Li S, et al. Changes in transcriptome profiling during the acute/subacute phases of contusional spinal cord injury in rats. Ann Transl Med 2020;8:1682.

17. Brazda N, Estrada V, Voss C, et al. Experimental Strategies to Bridge Large Tissue Gaps in the Injured Spinal Cord after Acute and Chronic Lesion. J Vis Exp 2016;(110):e53331.

18. Picoli CC, Coimbra-Campos LMC, Guerra DAP, et al. Pericytes Act as Key Players in Spinal Cord Injury. Am J Pathol 2019;189:1327-37.

19. An H, Li Q, Wen J. Bone marrow mesenchymal stem cells encapsulated thermal-responsive hydrogel network bridges combined photo-plasmonic nanoparticulate system for the treatment of urinary bladder dysfunction after spinal cord injury. J Photochem Photobiol B 2020;203:111741.

20. Wu C, Bao G, Xu G, et al. Triad1 regulates the expression and distribution of EHD1 contributing to the neurite outgrowth of neurons after spinal cord injury. J Cell Biochem 2019;120:5355-66.

21. Shepard JA, Stevans AC, Holland S, et al. Hydrogel design

Cite this article as: Zhang $\mathrm{H}$, Xu J, Saijilafu. The effects of GelMA hydrogel on nerve repair and regeneration in mice with spinal cord injury. Ann Transl Med 2021;9(14):1147. doi: 10.21037/atm-21-2874 for supporting neurite outgrowth and promoting gene delivery to maximize neurite extension. Biotechnol Bioeng 2012;109:830-9.

22. Fan L, Liu C, Chen X, et al. Directing Induced Pluripotent Stem Cell Derived Neural Stem Cell Fate with a ThreeDimensional Biomimetic Hydrogel for Spinal Cord Injury Repair. ACS Appl Mater Interfaces 2018;10:17742-55.

23. Vagner T, Dvorzhak A, Wojtowicz AM, et al. Systemic application of AAV vectors targeting GFAP-expressing astrocytes in Z-Q175-KI Huntington's disease mice. Mol Cell Neurosci 2016;77:76-86.

24. Wang T, Choi E, Monaco MC, et al. Direct induction of human neural stem cells from peripheral blood hematopoietic progenitor cells. J Vis Exp 2015;(95):52298.

25. Chen X, Wu H, Feng J, et al. Transcriptome profiling unveils GAP43 regulates ABC transporters and EIF2 signaling in colorectal cancer cells. BMC Cancer 2021;21:24.

26. Li X, Wang X, Chen H, et al. A comparative study of the behavior of neural progenitor cells in extrusion-based in vitro hydrogel models. Biomed Mater 2019;14:065001. 